\title{
Speech segmentation with a neural encoder model of working memory
}

\author{
Micha Elsner and Cory Shain \\ melsner0@gmail.com and shain.3@osu.edu \\ Department of Linguistics \\ The Ohio State University, Columbus, OH 43210
}

\begin{abstract}
We present the first unsupervised LSTM speech segmenter as a cognitive model of the acquisition of words from unsegmented input. Cognitive biases toward phonological and syntactic predictability in speech are rooted in the limitations of human memory (Baddeley et al., 1998); compressed representations are easier to acquire and retain in memory. To model the biases introduced by these memory limitations, our system uses an LSTMbased encoder-decoder with a small number of hidden units, then searches for a segmentation that minimizes autoencoding loss. Linguistically meaningful segments (e.g. words) should share regular patterns of features that facilitate decoder performance in comparison to random segmentations, and we show that our learner discovers these patterns when trained on either phoneme sequences or raw acoustics. To our knowledge, ours is the first fully unsupervised system to be able to segment both symbolic and acoustic representations of speech.
\end{abstract}

\section{Introduction}

This paper describes a new cognitive model of the acquisition of word-like units from unsegmented input. The model is intended to describe the process by which pre-linguistic infants learn their earliest words, a stage they pass through during the first year of life (Jusczyk and Aslin, 1995; Bergelson and Swingley, 2012). Our model is based on the standard memory model of Baddeley and Hitch (1974) in which the listener encodes lexical items into phonological working memory, but represents the entire sentence as a higher-level syn- tactic structure without phonological detail. Our model implements this architecture using encoderdecoder LSTMs with limited memory capacity, then searches for word segmentations which make it easy to remember the sentence. ${ }^{1}$

Word learning has been extensively studied in previous research, both with transcribed symbolic input and acoustics. Why attempt yet another approach? Our model has three main advantages. First, as a cognitive model, it relates the kinds of learning biases used in previous work to the wider literature on working memory. Second, its sequence-to-sequence neural architecture allows it to handle either one-hot symbolic input or dense vectors of acoustic features. In contrast, existing models are typically designed for "clean" symbolic input, then retrofitted with additional mechanisms to cope with acoustics. Finally, neural networks have been impressively successful in supervised language processing domains, yet are still underused in unsupervised learning. Even systems which do use neural nets to model lexical acquisition generally require an auxiliary model for clustering the embeddings, which can make their learning objectives difficult to understand. Our system uses the well-understood autoencoder objective to perform the segmentation task without requiring auxiliary clustering, and thus suggests a new direction for neural unsupervised learning.

In an experiment conducted on the widely used Brent corpus (Brent, 1999), our system achieves performance close to that of Fleck (2008), although subsequent systems outperform ours by a wider margin. We show that memory limitations do indeed drive the performance of the system, with smaller LSTM hidden states outperforming larger ones in the development set.

In a follow-up experiment designed to ex-

\footnotetext{
${ }^{1}$ The system is available from https://github. $\mathrm{com} / \mathrm{melsner/neural-segmentation.}$
} 
plore the flexibility of our model, we deploy the segmenter on acoustic input: the English portion of the Zerospeech 2015 challenge (Versteegh et al., 2015). Our model outperforms the winning model from that challenge (Räsänen et al., 2015), although we underperform more recent unsupervised acoustic segmentation systems (Kamper et al., 2016; Räsänen et al., under review).

To our knowledge, our system is the first unsupervised LSTM speech segmenter, as well as the first unsupervised speech segmenter to succeed on both symbolic and acoustic representations of speech. Our results are of note for several reasons. First, they provide modeling support for the claim that memory limitations encourage lexical acquisition. Second, they show that a general strategy of searching for maximally compressible representations can realistically guide lexical acquisition without explicit reference to perceptual biases (c.f. e.g. Räsänen et al., 2015), regardless of input representation. And third, they demonstrate the benefits of our adaptation of neural sequence modeling to unsupervised learning.

\section{Motivations}

We begin with a short overview of previous approaches to the word learning problem, then explain each of our main contributions in detail. Many cognitive models of the word learning problem draw on Brent (1999), which used a simple unigram model of the lexicon to discover repeated patterns in phonemically transcribed input. Brent's model laid the groundwork for later generative models with more sophisticated prior distributions over word frequencies, co-occurrence statistics and phonological shapes (Johnson and Goldwater, 2009, among others). Other modeling architectures for segmentation have focused on detecting phonological boundaries between words using transitional probabilities (Christiansen et al., 1998, among others) or inducing words procedurally by "subtracting" known word forms from utterances (Lignos, 2011).

All these modeling architectures are designed to work with phonemically transcribed input, and require some degree of retrofitting to work with more realistic inputs. In the Bayesian framework, this typically takes the form of a transducer which probabilistically transforms "underlying" lexical items to "surface" acoustics (Lee et al., 2015) or discrete symbols (Elsner et al., 2013); the same framework is used for morphological segmentation in Cotterell et al. (2015). For transitionbased models, the input must be transformed into discrete symbols from which segment-to-segment probabilities can be extracted; this transformation requires an externally trained preprocessor (a phone recognizer). Transition-based models are fairly robust to variation in the symbols (Rytting, 2007; Rytting et al., 2010; Daland and Pierrehumbert, 2011; Fleck, 2008) and can be relatively successful in this framework. Extensions using neural nets (Christiansen et al., 1998; Rytting et al., 2010) are discussed in more detail below (subsec. 2.3). Lignos (2011) requires the most complex preprocessing of the input (segmentation into syllables, with marked lexical stresses); adapting it to noisy input is an open problem.

\subsection{Working memory and learning biases}

Cognitive models of word segmentation rely on two kinds of learning biases to structure their inferred lexicons: predictability within words (often expressed as a prior over phonological forms), and Zipfian unigram and bigram frequencies of words (a prior over word distributions). These biases control the entropy of utterances, making it easy for adult listeners to remember what they hear and reconstruct any missing parts from context (Piantadosi et al., 2012). The biases correspond to different components in a standard model of working memory (Baddeley, 2007; Baddeley and Hitch, 1974). In this model, listeners can store the last few items they heard in a phonological loop, from which words are transferred into episodic memory which represents them at a syntactic/semantic level.

Baddeley et al. (1998) claim that the phonological loop functions in word learning as well as processing by proficient listeners, aiding in the acquisition of unfamiliar words. They summarize a number of studies showing that the vocabulary size of typically developing infants correlates with their ability to remember a sequence of phonologically plausible non-words, a test of phonological loop capacity. Children with Specific Language Impairment, meanwhile, remember nonwords poorly, a deficit which may contribute to their atypically small vocabularies. Baddeley et al. (1998) argue that the ability to remember an unfamiliar phonological form in the short term is essential if it is to be transferred to long-term mem- 
ory as a datapoint for lexical learning. This account of word learning is one of a growing number which attempt to unify acquisition and speech processing in terms of the same real-time, resourceconstrained mechanisms (Apfelbaum and McMurray, 2016).

In our model, memorization itself can be viewed as the objective for early word learning. The model attempts to reconstruct its input from memory; chunks that are easy to reconstruct (and that make the context reconstructible) are good candidate words. The working memory model accounts for the two types of bias normally found in Bayesian segmenters. Phonological predictability due to consistent word shapes (Börschinger and Johnson, 2014; Johnson and Goldwater, 2009) reduces the load on the phonological loop. Predictability between words reduces the load on syntactic memory. The two memory systems draw on different cognitive resources, which correspond to different parameters of the model.

\subsection{Input representations}

As stated above, traditional segmentation models operate on phonemic transcriptions and must be adapted to cope with phonetic or acoustic input. For models which infer an explicit lexicon (i.e., those which do not simply count segment transitions), this takes the form of a mapping between the data and the space of "underlying" latent word forms.

Learning such a mapping can be problematic. Traditional generative learning models use parametric distributions over the data - for acoustics, Gaussians (Vallabha et al., 2007; Feldman et al., 2009) or Gaussian-HMMs (Lee and Glass, 2012; Lee et al., 2015). But these are a notoriously poor fit to real speech sounds (Glass, 2003).

An example of an alternative approach to representation learning from acoustics is Räsänen et al. (2015). They exploit known acoustic indicators of syllable boundaries to infer syllable segments, cluster those segments using expectationmaximization (EM), and then identify multisyllabic words by searching for recurring cluster $n$ grams. As a result, their system is constrained to propose word boundaries only at proposed syllable boundaries regardless of the representations acquired downstream. Furthermore, EM is known to find non-optimal solutions for many problems in natural language (Johnson, 2007). To the ex- tent that this inhibits their system's ability to exploit information in the acoustic feature space, it might lead to misidentification of recurrent syllable $n$-grams and consequently to segmentation error.

Latent underlying representations can also cause search problems, since the model must explore all the possible underlying forms which might map to some utterance on the surface. In a probabilistic system capable of mapping every word to every possible realization, this quickly becomes intractable. Many systems use dynamic programming (Mochihashi et al., 2009; Neubig et al., 2010), sometimes with pruning (Van Gael et al., 2008). But these algorithms require Markov models with small context windows, and in any case can still be slow and prone to search errors.

Neural nets, on the other hand, learn a nonlinear mapping between input and output. This allows them to model speech more flexibly, outcompeting Gaussian/HMMs for supervised speech recognition (Graves et al., 2013; Hinton et al., 2012). Recurrent neural nets also produce hidden representations differently than HMMs. Rather than use dynamic programming to search a latent space, they produce a single vector deterministically at each timestep. Models such as LSTMs (Hochreiter and Schmidhuber, 1997) can learn long-distance sequential dependencies in their input without making inference more expensive.

\subsection{Neural unsupervised learning}

A few previous papers have used neural networks for word segmentation. Christiansen et al. (1998), drawing on older work with Simple Recurrent Networks (Elman, 1990), trains a recurrent network as a language model. Word boundaries are extracted at points where the network predicts an upcoming utterance boundary; that is, utterance boundaries are used as distant supervision for the locations of word boundaries. While effective, this system uses symbolic rather than acoustic input. Moreover, it may have trouble with word endings which do not end utterances, such as the endings of function words; experiments show that infants learn detailed representations of function words by 13 months (Shi et al., 2006) and use known words as "anchors" for segmentation within utterances (Bortfeld et al., 2005).

Rytting (2007) adapts the Christiansen model to variable input by using the posterior probabil- 
ity distribution from a phone recognizer as its feature representation. This system was run on natural data; results for word boundary detection were significantly above chance, though still much less accurate than results for symbolic input. The use of utterance boundaries as distant supervision may create problems for this system similar to those pointed out for Christiansen above. Moreover, the use of an SRN rather than an LSTM means that the system is essentially phonotatic; it makes its decisions based on the previous one or two phones, without the capacity to remember whole lexical items.

Recent work (Kamper et al., 2016) has attempted to harness the flexibility of neural feature extractors within the generative model framework. This model has a hybrid architecture consisting of a neural feature extractor, the Correspondence Autoencoder, pretrained using distant supervision (Kamper et al., 2015), and a Bayesian clustering/segmentation model. The system represents each word by neurally encoding its frames, then downsampling to obtain a fixed-dimensional word vector; the clustering model assumes that these vectors can be modeled with Gaussian clusters. The advantage of this approach is its ability to exploit the known strengths of both Bayesian and neural learning systems. The disadvantage is its indirectness: there is no end-to-end objective to be optimized, and the system's lexical learning does not inform its phonetic representations.

Even outside the domain of segmentation, neural networks have been most successful for supervised problems, and are not widely used for unsupervised learning of discrete structures (trees, clusters, segment boundaries). While some researchers have proposed information-theoretic objectives for learning clusters (Klapper-Rybicka et al., 2001), the most widely used unsupervised objective is the one used here: autoencoding. Yet autoencoders are rarely used to learn discrete hidden structures. One exception, Socher et al. (2011), uses autencoders to find a latent tree structure for sentiment analysis by greedily merging adjacent nodes so as to minimize the reconstruction error.

Chung et al. (2017) describe a model similar to our own which performs a segmentation task using autoencoders. Both models use multiscale autoencoding to learn a sequence model with unknown segment boundaries. The main difference is the different technique used to deal with the discontinuities caused by switching discrete segment boundary variables. However, they evaluate their model on downstream tasks (notably, character language modeling) without evaluating the segmentations directly.

\section{The Model}

The model uses a basic encoder-decoder architecture now typical in machine translation (Cho et al., 2014) and image captioning (Vinyals et al., 2015). In a typical encoder-decoder, the input is fed into an LSTM sequence model (Hochreiter and Schmidhuber, 1997) which represents it as a latent numeric embedding. This embedding is then fed into another sequence model, which uses it to generate an output sequence. Our two-level model performs this process in stages, first encoding every word, character-by-character, and then encoding the word sequence, vector-by-vector. In an autoencoder, the objective is to make input and output match; thus, the decoder performs the encoding stages in reverse. We provide the final encoder hidden state as input to each decoder unit. To force the system's learned embeddings to be robust to noise caused by mishearing or misremembering, we use dropout (Srivastava et al., 2014) at the input (deleting individual timesteps) and at the word encoding layer (deleting entire words). This architecture is illustrated in Figure 1.

The encoder-decoder does not predict segment boundaries directly, but gives an objective function (reconstruction loss) which can be used to guide segmentation. Because the segment boundary decisions are hard (there are no "partial" boundaries), the loss function is not differentiable as a function of the boundary indicators. We use sampling to estimate the gradient, as in previous work (Mnih et al., 2014; Xu et al., 2015). Our sampling system works as follows: we begin with a proposal distribution $P_{\text {seg }}$ over sequences of segment boundaries for the current utterance $x$. We sample $m$ sequences of boundaries, $B_{1: m}$ from $P_{\text {seg }}$. Each boundary sequence splits the utterance into words. We use the autoencoder network to encode and decode the words, and obtain the loss (the cross-entropy of the reconstructed input) for each sequence, $L_{1: m}$.

We can use the cross-entropy to estimate the posterior probability of the data given a breakpoint sequence (Eq. 1), assuming a uniform prior over 


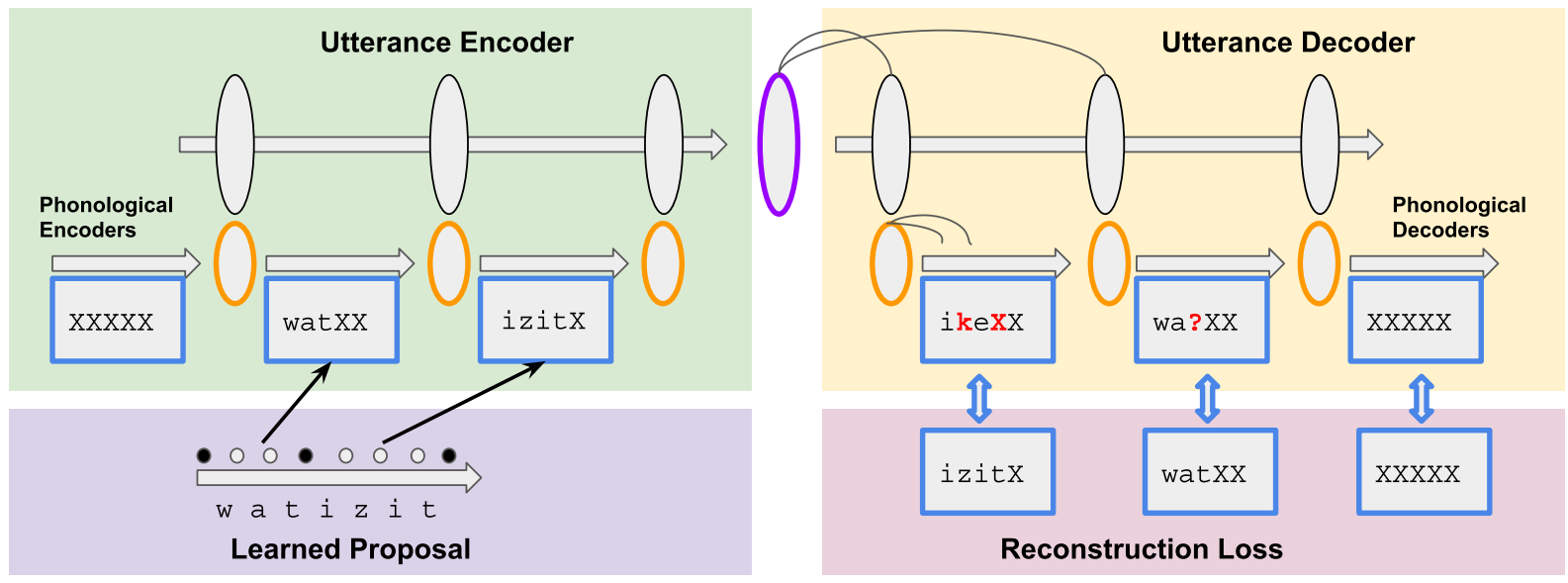

Figure 1: Architecture of the model: top two panels show the encoder/decoder, bottom panels show computation of breakpoints and resulting loss. Horizontal arrows represent LSTMs.

break positions. We then treat each breakpoint $t$ in the utterance independently: for each one, we use the losses and the proposal probabilities to compute an importance weight $w_{i}^{t}$ for sample $i$ and position $t$ (Eq. 2), then compute the expected probability of a boundary at that position by summing over the weighted samples (Eq. 3). Essentially, a breakpoint will be more likely if it appeared in samples with low reconstruction loss, especially if it is not encouraged by the current proposal.

$$
\begin{aligned}
P\left(x \mid B_{i}\right) & =\frac{P\left(B_{i} \mid x\right) P\left(B_{i}\right)}{P(x)} \approx \frac{\exp \left(L_{i}\right)}{\sum_{j} \exp \left(L_{j}\right)} \\
w_{i}^{t} & =\frac{P\left(x \mid B_{i}\right)}{P_{\text {seg }}^{t}\left(B_{i}^{t}\right)} \\
\mathbb{E}[B(t)] & \approx \frac{1}{\sum_{i} w_{i}^{t}} \sum_{i} w_{i}^{t} B_{i}^{t}
\end{aligned}
$$

We initialize by making random breakpoint proposals (with probability .1 at each position). The random proposal does not search the space of segmentation boundaries particularly efficiently, so we train a better proposal using another LSTM. This LSTM simply reads the input from left to right and predicts a binary output (segment or not) at each timestep. We update the proposal LSTM by using the sampling-derived $P_{\text {seg }}$ as a training target after each batch. Thus, the proposal learns to predict segment boundaries that are likely to result in low reconstruction loss for the main network. To force the system to explore the space, we smooth the learned proposal by interpolating it with a uniform distribution: $P_{\text {seg }}=.9 \times P_{L S T M}+$ $.1 \times \frac{1}{2}$.
We control the memory capacity of the system using four tunable parameters: the number of hidden states at the phonological level $\left(H_{p}\right)$ and at the utterance level $\left(H_{u}\right)$ and the dropout probability of mishearing a phonological segment $\left(D_{p}\right)$ or a word $\left(D_{u}\right)$. We discuss parameter tuning results below.

The system also has several other parameters which were not tuned against the evaluation metric. For convenience in GPU training, we treat all sequences as fixed length, either clipping them or padding with a dummy symbol. This requires us to set a maximum length for each word (in characters), and each utterance (in words and characters); we set these parameters to ensure $99 \%$ coverage of the input (for the Brent corpus, 7, 10, and 30 respectively).

Clipping creates the possibility of pathological outcomes where the system deliberately creates extremely long words, exploiting the fact that the excess characters will be discarded and will not have to be predicted in the output. We penalize this by subtracting 50 for each deleted character. Finally, we find that, despite pre-training, the system may settle into an initial state where the phonological network simply embeds the characters and the utterance network learns a character LM. To avoid this, we subtract 10 from the objective for each one-symbol word. These parameters were tuned only lightly; we increased the values until the problematic behavior (segmentation of the entire utterance as one word, or each character as a word) ceased.

We implemented the network in Keras (Chollet, 2015), using Adam (Kingma and Ba, 2014) with 
default settings for optimization. We use minibatches of 128 and take 100 samples of potential segment boundaries per sequence. We perform 10 iterations of pretraining with random boundaries, 10 iterations of boundary induction with random proposals, and 70 iterations of full training with the learned LSTM proposal.

\section{Results}

\subsection{Brent Corpus}

The Brent corpus (Brent, 1999) is a standard benchmark dataset for segmentation, consisting of 9790 utterances from Bernstein-Ratner (1987), translated into phonemic transcription using the CMU dictionary. The standard metrics for segmentation are F-score for word boundary detection (treating each boundary in isolation) and Fscore for word token segmentation (a word is correct only if both its boundaries are correct and no spurious boundaries intervene). Although early work on Brent used all 9790 utterances for both development and test, we use the first 8000 utterances for parameter tuning. Thus, we present results for the whole corpus (for comparison with previous work) and clean test results for the last 1790.

We tune the four parameters of our system, $H_{p}, H_{u}, D_{p}$ and $D_{u}$, using a grid search (see Figure 2). Each subplot shows a particular dropout setting, $D_{p} / D_{u}$; the cells within represent settings of $H_{p}$ (rows) and $H_{u}$ (columns), where darker cells have higher boundary F-score. Excessive noise decreases scores, especially high word dropout (right side of the plot). For low levels of dropout, the best systems tend to have small numbers of hidden units (dark regions in the lower left); for larger dropout, more hidden units can be useful. For instance, compare the top left subplot, with 0 dropout and good performance with $H_{p}=$ $20, H_{u}=100$, to subplot 3,3, with optimal performance at $H_{p}=80, H_{u}=200$. In other words, limiting the system's memory resources is indeed the key to its performance. The best score occurs at $H_{p}=80, H_{u}=400, D_{p}=0.5, D_{u}=0.25$ with a dev boundary F-score of $83 \%$. We used these parameters for our final evaluation, along with 100 hidden units in the proposal network.

To further demonstrate that limited memory can bias the network to learn a low-entropy lexicon, we perform a separate experiment using the phonological encoder/decoder alone. We create
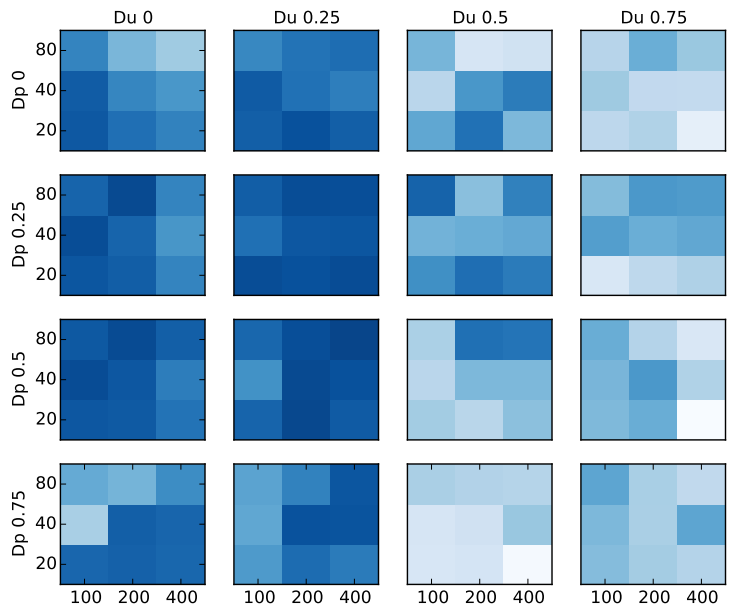

Figure 2: Tuning results on Brent development. Cell axes represent $H_{u}$ and $H_{p}$, darker cells have higher scores (best $83 \%$, worst $60 \%$ ).

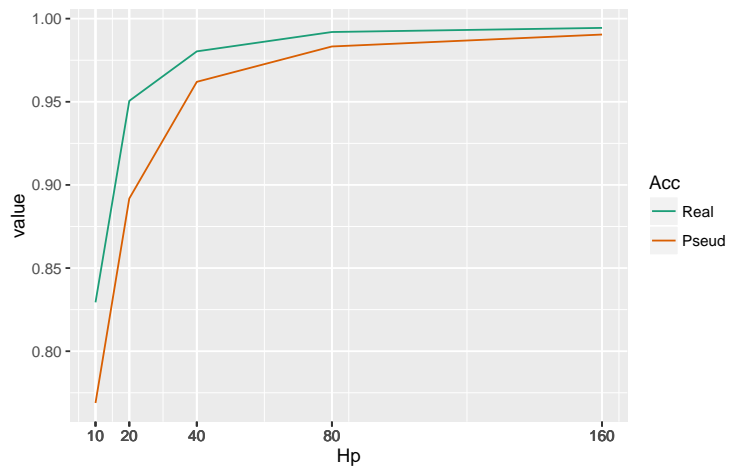

Figure 3: Reconstruction accuracy of the phonological encoder/decoder on real words vs. lengthmatched pseudowords from Brent.

networks with varying $H_{p}$ (setting $D_{p}$ to 0 ); for each network size, we train one net on real words from the gold segmentation of Brent, and another on length-matched pseudowords sampled by randomly segmenting the Brent corpus. Figure 3 shows the reconstruction error rates as a function of $H_{p}$. The gap between the green and orange lines shows the difference in reconstruction error obtained by using real words rather than pseudowords. For the smallest $H_{p}$, neither network does a good job; for the largest, both networks learn the sequences perfectly. For values in between, however, the lines are relatively far apart, showing that the real words are easier for the network to remember.

Our results for Brent, along with selected comparisons, are shown in Table $1 .^{2}$ Our system per-

\footnotetext{
${ }^{2}$ Comparison system scores are those reported in their
} 


\begin{tabular}{lcccc} 
System & Bd P & Bd R & Bd F & Wd F \\
\hline Goldwater 09 & 90 & 74 & 87 & 74 \\
Johnson 09 & - & - & - & 88 \\
Berg- & - & - & - & 88 \\
Kirkpatrick & & & & \\
10 & & & & \\
Fleck 08 & 95 & 74 & 83 & 71 \\
\hline Ours (all) & 81 & 85 & 83 & 72 \\
Ours (test) & 81 & 86 & 83 & 72
\end{tabular}

Table 1: Selected segmentation results on Brent.

forms at the lower end of the reported range for Brent segmenters, scoring $83 \%$ for boundary detection and $72 \%$ for word detection (comparable to (Fleck, 2008)). (Lignos (2011) scores 93\% boundary $\mathrm{F}$ on a different corpus with marked syllable boundaries.) From a cognitive modeling point of view, it is not clear what performance we should expect on Brent to model the performance of a young human infant. Models of early word segmentation are motivated by studies showing that, by their first birthday, infants can distinguish many common words from nonwords (Vihman et al., 2004; Swingley, 2005). But this does not imply that they learn every word they hear, or that they can use their word knowledge to segment every utterance correctly. Thus, while our result is not state-of-the-art, it is good enough to conform with the reported infant results and suggest that our neural architecture is a promising direction.

Learning curves for segmentation on the Brent corpus are shown in Figure 4. The first 10 iterations show a gradual increase in segmentation performance using the random proposal. Performance increases sharply with the activation of the learned proposal, then climbs slowly over time. Precision initially exceeds recall (that is, the system proposes too few boundaries) but recall climbs over time as the system exploits known words as "anchors" to discover new ones, a pattern consistent with the infant data (Bortfeld et al., 2005).

\subsection{Zerospeech 2015 acoustic segmentation}

We began by claiming that an advantage of our model was its flexible architecture that permits dense acoustic features as input (rather than symbolic phone labels) with little modification. In this section, we present preliminary results from

respective publications, except for Goldwater et al. (2009), which are corrected numbers published with their software release. Not all systems report all metrics.

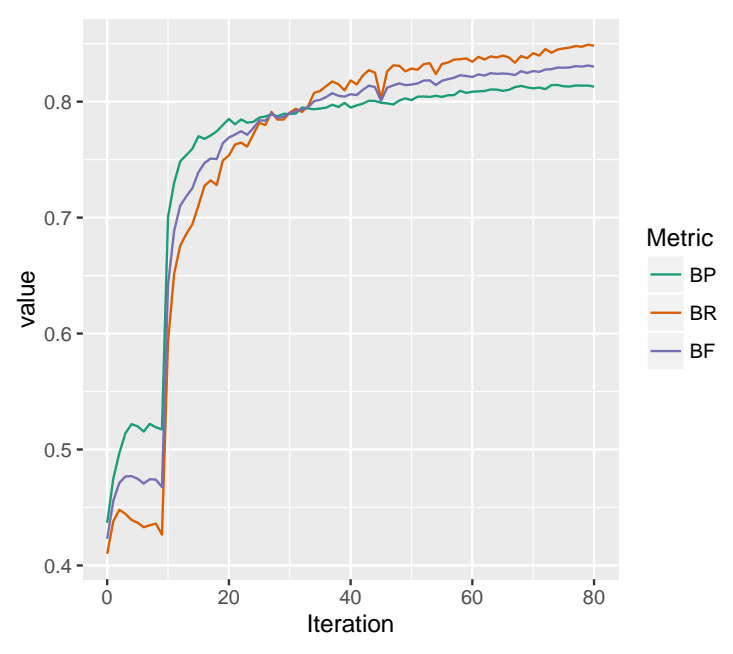

Figure 4: Boundary precision, recall, and F1 score by iteration on the entire Brent dataset.

a follow-up experiment in which we tested this claim by deploying our system as an acoustic segmenter on the English portion of the Zerospeech 2015 challenge dataset (Versteegh et al., 2015). We preprocess the raw acoustic data by extracting 25ms 13-dimensional mel frequency cepstral coefficients with first and second order deltas at a step size of $10 \mathrm{~ms}$. We then train the network on the resulting sequences of 39-dimensional frames.

Given that the goal of the experiment was to test the existing architecture on a novel task, we intentionally conducted this experiment with minimal parameter tuning or architectural modification. However, we made several key changes in response to the unique challenges presented by acoustic input.

First, since we are now reconstructing dense vectors of acoustic features, we use mean squared error (MSE) instead of categorical cross-entropy as the autoencoder loss function. We consequently rescale our clipping penalty from 50 to 1 , a coefficient which seemed more in balance with the variation in decoder loss produced by MSE. We also increase our one-letter penalty from 10 to 50 , modeling our strong prior assumption that a 1 -frame segment will never correspond to a word.

Second, in contrast to the phoneme sequences in the Brent corpus discussed above, utterance boundaries are not observed in acoustic input. The input to the two-level autoencoder must be divided into sequences of utterances, so we imposed utterance boundaries by iteratively consuming the next discovered word in the time series up to the maxi- 


\begin{tabular}{lcccc} 
System & $\mathrm{Bd} \mathrm{P}$ & $\mathrm{Bd} \mathrm{R}$ & $\mathrm{Bd} \mathrm{F}$ & $\mathrm{Wd} \mathrm{F}$ \\
\hline Lyzinski 15 & 18.8 & 64.0 & 29.0 & 2.4 \\
Räsänen 15 & 75.7 & 33.7 & 46.7 & 9.6 \\
Räsänen new & 61.1 & 50.1 & 55.2 & 12.4 \\
Kamper 16 & 66.5 & 58.8 & 62.4 & 20.6 \\
\hline Ours & 62.4 & 43.2 & 51.1 & 9.3
\end{tabular}

Table 2: Selected word segmentation results on the the Zerospeech 2015 English corpus.

mum utterance length (in frames). The aforementioned clipping penalties punish the system for utterances that contain too many words, preventing it from optimizing its autoencoder loss by segmenting everywhere.

Third, for our initial proposal distribution we use the speech region segmentation provided by the Zerospeech challenge, consisting of speech intervals identified through automatic voice activity detection (VAD), rather than using the uniform initialization described above for symbolic mode. We interpolate the initial distribution with a uniform prior as described above.

Fourth, we discovered in practice that the assumption of independence between samples made by the importance scoring scheme as implemented for symbolic mode was distortionary in acoustic mode, such that the "best" segmentation discovered through sampling often contained many times more segments than any of its component samples. ${ }^{3}$ To prevent this from happening, we simply used 1-best rather than importance sampling for acoustic segmentation.

We trained the system for 80 iterations using parameters $H_{p}=20, H_{u}=400, D_{p}=0, D_{u}=$ 0.25 and 1500 hidden units in the proposal LSTM. In the auto-encoder network, we limited frames per utterance, words per utterance, and frames per word to 400, 16, and 100, respectively. Results are presented in Table 2, along with a comparison to results from other systems. Lyzinski et al. (2015) and Räsänen et al. (2015) were entrants in the Zerospeech 2015 challenge, in which Räsänen et al. (2015) performed best in the word bound-

\footnotetext{
${ }^{3}$ We believe this is driven by training batches in which multiple samples receive similar scores but have fairly nonoverlapping segmentations. In this case, the output segmentation can contain something close to the union of the best samples' segmentation points, leading to oversegmentation. This effect is likely exaggerated in acoustic mode as compared to symbolic mode because acoustic word segments are generally much longer (in frames) than their corresponding symbolic word segments (in characters).
}

ary detection measure. As shown in the table, our system beats both of these competitors' boundary detection scores, with a word detection score comparable to that of Räsänen et al. (2015). However, since the challenge concluded, Räsänen et al. (under review) have modified their system and improved their segmentation score, ${ }^{4}$ and Kamper et al. (2016) have established a new state of the art for this task. While our system currently remains far from these newer benchmarks, we expect that with systematic parameter tuning and investigation into appropriate sampling procedures for acoustic input, we might be able to improve substantially on the results presented here. We believe that the results of this preliminary investigation into the acoustic domain are promising, and that they bear out our claims about the flexibility of our general architecture.

\section{Conclusions and future directions}

This work presented a new unsupervised LSTM architecture for discovering meaningful segments in representations of continuous speech. Memory limitations in the autoencoder part of the network apply pressure to discover compressed representations much as human memory limitations have been argued to guide lexical acquisition. By varying the size of the LSTM's hidden state, we showed that word segmentation performance on the Brent corpus is driven by memory limitations, with performance improving (up to a point) as we constrain the system's memory capacity. And by successfully deploying our system on both symbolic (character) and acoustic representations of speech, we demonstrated that our approach is flexible enough to adapt to either representation of the speech stimulus.

In the future we hope to pursue a number of lines of inquiry. We plan to conduct more detailed parameter tuning in the acoustic domain and to segment the Xitsonga dataset supplied with the Zerospeech 2015 challenge. We also intend to introduce additional layers into the autoencoder network so as to allow for joint acquisition of phone-like, morph-like, and/or word-like units in the acoustic signal; this may benefit from the alternate model structure of Chung et al. (2017). And we plan to explore clustering techniques that

\footnotetext{
${ }^{4}$ The new results are not yet published. Those reported above are copied from the results summary in Kamper et al. (2016).
} 
would allow our system to discover categories in addition to probable segmentation points.

\section{Acknowledgments}

We thank members of the Clippers discussion group (especially Joo-Kyung Kim, Eric FoslerLussier and Wei $\mathrm{Xu}$ ) and three anonymous reviewers. Computations for this project were run on a Titan-X GPU donated by the NVIDIA Hardware Grant program and on the Ohio Supercomputer (1987). Funding was provided by NSF \#1422987.

\section{References}

Keith S Apfelbaum and Bob McMurray. 2016. Learning during processing: Word learning doesn't wait for word recognition to finish. Cognitive Science.

Alan Baddeley. 2007. Working memory, thought and action. Oxford University Press, Oxford, UK.

Alan Baddeley, Susan Gathercole, and Costanza Papagno. 1998. The phonological loop as a language learning device. Psychological review 105(1):158.

Alan Baddeley and Graham Hitch. 1974. Working memory. Psychology of learning and motivation 8:47-89.

Elika Bergelson and Daniel Swingley. 2012. At 69 months, human infants know the meanings of many common nouns. Proceedings of the National Academy of Sciences 109(9):3253-3258.

Nan Bernstein-Ratner. 1987. The phonology of parentchild speech. In K. Nelson and A. van Kleeck, editors, Children's Language, Erlbaum, Hillsdale, NJ, volume 6.

Benjamin Börschinger and Mark Johnson. 2014. Exploring the role of stress in Bayesian word segmentation using adaptor grammars. Transactions of the Association for Computational Linguistics 2:93104.

Heather Bortfeld, James L. Morgan, Roberta Michnick Golinkoff, and Karen Rathbun. 2005. Mommy and me. Psychological Science 16(4):298-304. https://doi.org/10.1111/j.0956-7976.2005.01531.x.

Michael R. Brent. 1999. An efficient, probabilistically sound algorithm for segmentation and word discovery. Machine Learning 34:71-105.

Ohio Supercomputer Center. 1987. Ohio supercomputer center. http://osc.edu/ark: /19495/f5s1ph73.

Kyunghyun Cho, Bart van Merrienboer, Caglar Gulcehre, Dzmitry Bahdanau, Fethi Bougares, Holger Schwenk, and Yoshua Bengio. 2014. Learning phrase representations using RNN encoder-decoder for statistical machine translation. In Proceedings of the 2014 Conference on Empirical Methods in Natural Language Processing (EMNLP). Association for Computational Linguistics, Doha, Qatar, pages 1724-1734. http://www.aclweb.org/anthology/D141179.

François Chollet. 2015. Keras. https : / / github. $\mathrm{com} / \mathrm{fchollet/keras.}$

Morten H. Christiansen, Joseph Allen, and Mark S. Seidenberg. 1998. Learning to segment speech using multiple cues: A connectionist model. Language and Cognitive Processes 13(2/3):221-269.

Junyoung Chung, Sungjin Ahn, and Yoshua Bengio. 2017. Hierarchical multiscale recurrent neural networks. In Proceedings of ICLR.

Ryan Cotterell, Nanyun Peng, and Jason Eisner. 2015. Modeling word forms using latent underlying morphs and phonology. Transactions of the Association for Computational Linguistics 3:433-447.

Robert Daland and Janet B. Pierrehumbert. 2011. Learning diphone-based segmentation. Cognitive Science 35(1):119-155. https://doi.org/10.1111/j.1551-6709.2010.01160.x.

Jeffrey L Elman. 1990. Finding structure in time. Cognitive science 14(2):179-211.

Micha Elsner, Sharon Goldwater, Naomi Feldman, and Frank Wood. 2013. A joint learning model of word segmentation, lexical acquisition, and phonetic variability. In Proceedings of the 2013 Conference on Empirical Methods in Natural Language Processing. Association for Computational Linguistics, Seattle, Washington, USA, pages 4254. http://www.aclweb.org/anthology/D13-1005.

Naomi Feldman, Thomas Griffiths, and James Morgan. 2009. Learning phonetic categories by learning a lexicon. In Proceedings of the 31st Annual Conference of the Cognitive Science Society.

Margaret M. Fleck. 2008. Lexicalized phonotactic word segmentation. In Proceedings of ACL-08: HLT. Association for Computational Linguistics, Columbus, Ohio, pages 130-138. http://www.aclweb.org/anthology/P/P08/P08-1016.

James Glass. 2003. A probabilistic framework for segment-based speech recognition. Computer Speech and Language 17:137-152.

Sharon Goldwater, Thomas L. Griffiths, and Mark Johnson. 2009. A Bayesian framework for word segmentation: Exploring the effects of context. Cognition 112(1):21-54.

Alex Graves, Abdel-rahman Mohamed, and Geoffrey Hinton. 2013. Speech recognition with deep recurrent neural networks. In 2013 IEEE International Conference on Acoustics, Speech and Signal processing (ICASSP). IEEE, pages 6645-6649. 
Geoffrey Hinton, Li Deng, Dong Yu, George Dahl, Abdel rahman Mohamed, Navdeep Jaitly, Andrew Senior, Vincent Vanhoucke, Patrick Nguyen, Tara Sainath, and Brian Kingsbury. 2012. Deep neural networks for acoustic modeling in speech recognition. IEEE Signal Processing Magazine 28(6):8297.

Sepp Hochreiter and Jürgen Schmidhuber. 1997. Long short-term memory. Neural computation 9(8):1735-1780.

Mark Johnson. 2007. Why doesn't EM find good HMM POS-taggers? In EMNLP.

Mark Johnson and Sharon Goldwater. 2009. Improving nonparametric Bayesian inference: Experiments on unsupervised word segmentation with adaptor grammars. In Proceedings of Human Language Technologies: The 2009 Annual Conference of the North American Chapter of the Association for Computational Linguistics. Boulder, Colorado.

Peter W Jusczyk and Richard N Aslin. 1995. Infants' detection of the sound patterns of words in fluent speech. Cognitive psychology 29(1):1-23.

Herman Kamper, Micha Elsner, Aren Jansen, and Sharon Goldwater. 2015. Unsupervised neural network based feature extraction using weak top-down constraints. In Acoustics, Speech and Signal Processing (ICASSP), 2015 IEEE International Conference on. IEEE, pages 5818-5822.

Herman Kamper, Aren Jansen, and Sharon Goldwater. 2016. A segmental framework for fullyunsupervised large-vocabulary speech recognition. arXiv preprint arXiv:1606.06950 .

Diederik P. Kingma and Jimmy Ba. 2014. Adam: A method for stochastic optimization. CoRR abs/1412.6980. http://arxiv.org/abs/1412.6980.

Magdalena Klapper-Rybicka, Nicol N Schraudolph, and Jürgen Schmidhuber. 2001. Unsupervised learning in LSTM recurrent neural networks. In International Conference on Artificial Neural Networks. Springer, pages 684-691.

Chia-ying Lee and James Glass. 2012. A nonparametric Bayesian approach to acoustic model discovery. In Proceedings of the 50th Annual Meeting of the Association for Computational Linguistics (Volume 1: Long Papers). Association for Computational Linguistics, Jeju Island, Korea, pages 40-49. http://www.aclweb.org/anthology/P12-1005.

Chia-ying Lee, Timothy J O'Donnell, and James Glass. 2015. Unsupervised lexicon discovery from acoustic input. Transactions of the Association for Computational Linguistics 3:389-403.

Constantine Lignos. 2011. Modeling infant word segmentation. In Proceedings of the fifteenth conference on computational natural language learning. Association for Computational Linguistics, pages 29-38.
Vince Lyzinski, Gregory Sell, and Aren Jansen. 2015. An evaluation of graph clustering methods for unsupervised term discovery. In Proceedings of Interspeech 2015.

Volodymyr Mnih, Nicolas Heess, Alex Graves, and Koray Kavukcuoglu. 2014. Recurrent models of visual attention. In Advances in Neural Information Processing Systems. pages 2204-2212.

Daichi Mochihashi, Takeshi Yamada, and Naonori Ueda. 2009. Bayesian unsupervised word segmentation with nested Pitman-Yor language modeling. In Proceedings of the Joint Conference of the 47th Annual Meeting of the ACL and the 4th International Joint Conference on Natural Language Processing of the AFNLP. Association for Computational Linguistics, Suntec, Singapore, pages 100 108. http://www.aclweb.org/anthology/P/P09/P091012.

Graham Neubig, Masato Mimura, Shinsuke Mori, and Tatsuya Kawahara. 2010. Learning a language model from continuous speech. In 11th Annual Conference of the International Speech Communication Association (InterSpeech 2010). Makuhari, Japan, pages 1053-1056.

Steven T Piantadosi, Harry Tily, and Edward Gibson. 2012. The communicative function of ambiguity in language. Cognition 122(3):280-291.

Okko Räsänen, Gabriel Doyle, and Michael C. Frank. 2015. Unsupervised word discovery from speech using automatic segmentation into syllable-like units. In Proceedings of Interspeech 2015. pages 3204-3208.

Okko Räsänen, Gabriel Doyle, and Michael C. Frank. under review. Pre-linguistic rhythmic segmentation of speech into syllabic units.

Anton Rytting. 2007. Preserving Subsegmental Variation in Modeling Word Segmentation (Or, the Raising of Baby Mondegreen). Ph.D. thesis, The Ohio State University.

Anton Rytting, Chris Brew, and Eric Fosler-Lussier. 2010. Segmenting words from natural speech: subsegmental variation in segmental cues. Journal of Child Language 37(3):513-543.

Rushen Shi, Janet F Werker, and Anne Cutler. 2006. Recognition and representation of function words in english-learning infants. Infancy 10(2):187-198.

Richard Socher, Jeffrey Pennington, Eric H Huang, Andrew Y Ng, and Christopher D Manning. 2011. Semi-supervised recursive autoencoders for predicting sentiment distributions. In Proceedings of the conference on empirical methods in natural language processing. Association for Computational Linguistics, pages 151-161. 
Nitish Srivastava, Geoffrey E Hinton, Alex Krizhevsky, Ilya Sutskever, and Ruslan Salakhutdinov. 2014. Dropout: a simple way to prevent neural networks from overfitting. Journal of Machine Learning Research 15(1):1929-1958.

Daniel Swingley. 2005. Statistical clustering and the contents of the infant vocabulary. Cognitive Psychology 50:86-132.

Gautam K. Vallabha, James L. McClelland, Ferran Pons, Janet F. Werker, and Shigeaki Amano. 2007. Unsupervised learning of vowel categories from infant-directed speech. Proceedings of the National Academy of Sciences 104(33):13273-13278.

Jurgen Van Gael, Yunus Saatci, Yee Whye Teh, and Zoubin Ghahramani. 2008. Beam sampling for the infinite Hidden Markov model. In Proceedings of the 25th International Conference on Machine learning. ACM, New York, NY, USA, ICML '08, pages 1088-1095. https://doi.org/10.1145/1390156.1390293.

Maarten Versteegh, Roland Thiollière, Thomas Schatz, Xuan Nga Cao, Xavier Anguerra, Aren Jansen, and Emmanuel Dupoux. 2015. The zero resource speech challenge 2015. In Proceedings of Interspeech 2015 .

Marilyn M Vihman, Satsuki Nakai, Rory A DePaolis, and Pierre Hallé. 2004. The role of accentual pattern in early lexical representation. Journal of Memory and Language 50(3):336-353.

Oriol Vinyals, Alexander Toshev, Samy Bengio, and Dumitru Erhan. 2015. Show and tell: A neural image caption generator. In Proceedings of the IEEE Conference on Computer Vision and Pattern Recognition. pages 3156-3164.

Kelvin Xu, Jimmy L. Ba, Ryan Kiros, Kyunghyun Cho, Aaron Courville, Ruslan Salakhutdinov, Richard S. Zemel, and Yoshua Bengio. 2015. Show, attend and tell: Neural image caption generation with visual attention. In Proceedings of ICML. JMLR, Lille, France. 